\title{
SERVPRO: uma técnica para a gestão de operações de serviços
}

\author{
Luciano Costa Santos, M.Eng. \\ Programa de Pós-Graduação em \\ Engenharia de Produção - UFSC \\ E-mail: Icostas@eps.ufsc.br \\ Gregorio Varvakis, Ph.D. \\ CIN \& PPGEP - UFSC \\ E-mail: grego@eps.ufsc.br
}

\begin{abstract}
Resumo
Atualmente, a função operações tem merecido um destaque cada vez maior nas empresas de serviços. Entretanto, poucas técnicas utilizadas na gestão de operações de serviços têm uma abordagem específica para as caraterísticas dos serviços. Este artigo apresenta uma técnica de projeto e análise de processos que permite aos gerentes visualizarem os serviços na perspectiva do cliente. A técnica proposta (denominada SERVPRO), que é baseada na técnica IDEF3, considera as características específicas dos serviços e visa a melhoria da qualidade. Após uma breve revisão de algumas técnicas de projeto e análise de processos de serviços, a técnica proposta é apresentada.
\end{abstract}

\section{Palavras-chave}

Gestão de operações de serviços, Projeto e análise de processos, Qualidade em serviços.

\section{SERVPRO: a service operations management tool}

\begin{abstract}
The importance of the operations function in service organizations is on growth nowadays. However, few techniques of service operations management have a specific approach to the features of service processes. This paper aims to present a service design technique that allows managers to systematically design and analyze their service processes from a customer's perspective. The proposed technique (called SERVPRO), which is based on IDEF3 technique, considers the specific characteristics of service processes and focuses the service quality. Following a brief review of some techniques used in the design and analysis of service processes, the proposed technique is shown.
\end{abstract}




\section{INTRODUĈ̣̃O}

Um dos grandes desafios atuais para os profissionais de Engenharia de Produção é a aplicação dos princípios de gestão da produção em operações de serviços. De fato, a maior parte do conhecimento acumulado no campo da gestão da produção foi originado em ambientes de manufatura, sendo que muito pouco tem sido desenvolvido para operações de serviços. Porém, com a grande participação do setor de serviços na economia e com a agregação de serviços como diferencial competitivo em atividades de manufatura, a Gestão de Operações de Serviços torna-se um dos principais imperativos na área de gestão da produção (JOHNSTON, 1994).

Dentre as diferentes áreas de aplicação, o projeto de serviços destaca-se como um dos principais tópicos na agenda de pesquisa em Gestão de Operações de Serviços (JOHNSTON, 1999a). Para entender as peculiaridades no projeto de um serviço, é importante considerar que a sobreposição entre produto e processo nas operações de serviços é maior que nas operações de manufatura (SLACK et al., 1997). Fitzsimmons\&Fitzsimmons (1998) chegam a declarar que, em serviços, o produto é o processo. $\mathrm{O}$ fato é que o cliente de serviços faz parte do processo de transformação, o que torna difícil a separação entre projeto do produto e projeto do processo. Até mesmo os processos que não envolvem o cliente (processos de retaguarda) se sobrepõem ao "produto", pois dão suporte direto aos processos em que o cliente participa (processos de linha de frente). Assim, o projeto do processo assume uma importância primordial no projeto de serviços, pois é ele que vai determinar a natureza das interações (momentos da verdade) na produção do serviço.

$\mathrm{O}$ artigo "Designing services that deliver", escrito por Shostack (1984), foi um dos primeiros trabalhos introduzidos na literatura a enfatizar o projeto de serviços sob a perspectiva do processo. Nesse trabalho, a autora apresenta uma técnica de mapeamento de processos de serviços chamada service blueprint, que pode ser utilizada tanto no projeto como na análise do processo.

$\mathrm{Na}$ verdade, muitas das técnicas utilizadas para projetar processos de serviços podem ser aplicadas tanto no projeto de novos serviços como na análise do projeto de serviços existentes. De acordo com Gummesson (1994), como não existe uma tradição em se projetar novos serviços, freqüentemente é necessário que a metodologia de projeto de serviços também seja adequada para a análise dos processos existentes.

Em geral, as técnicas utilizadas no projeto de serviços visam representar os processos de serviços de forma a permitir o entendimento do processo, para projetar novos serviços ou analisar processos existentes. Contudo, não se pode afirmar que todas as técnicas utilizadas em projeto e análise de processos de serviços sejam adequadas para esse fim. Muitas das técnicas utilizadas não consideram as especificidades das operações de serviços e falham no suporte à melhoria da qualidade nos processos.

Para uma gestão eficaz das operações de serviços, é necessário conhecer os processos que produzem os serviços. Também é necessário considerar que o cliente participa do processo de produção de serviços. Com o entendimento do processo e o reconhecimento da participação do cliente no serviço, pode-se tratar a Gestão de Operações de Serviços de uma forma adequada. Caso contrário, existe um risco de que as técnicas de gestão da produção que foram bemsucedidas na manufatura sejam aplicadas sem êxito em operações de serviços. É importante lembrar que os processos de serviços exigem uma abordagem específica. Assim, as técnicas tradicionais de gestão da produção devem ser adaptadas à realidade das operações de serviços, ou até mesmo técnicas específicas devem ser criadas.

Este artigo apresenta uma técnica de projeto e análise de processos de serviços que traz uma abordagem específica para a Gestão de Operações de Serviços. O principal objetivo da técnica proposta nesse trabalho é fornecer suporte para a melhoria da qualidade em serviços, podendo também ser adaptada para diferentes aplicações. A técnica proposta foi baseada no IDEF3, uma técnica tradicionalmente utilizada nos processos de manufatura. Após as devidas adaptações, denominou-se a técnica resultante de SERVPRO, que mesmo não representando uma técnica inteiramente nova, apresenta importantes diferenças conceituais em relação ao IDEF3 original.

\section{TÉCNICAS DE PROJETO E ANÁLISE DE PROCESSOS DE SERVIC̣OS}

Desde o surgimento do service blueprint de Shostack (1984, 1987), algumas técnicas para projeto e análise de processos de serviços têm sido desenvolvidas. A maioria delas segue a lógica do service blueprint, que considera a participação do cliente nos processos de serviços.

Dentre as técnicas para projeto e análise de processos de serviços encontradas na literatura, o service blueprint representa a técnica mais conhecida. De acordo com Fitzsimmons \& Fitzsimmons (1998), o service blueprint é um mapa de todas as transações que constituem o processo de entrega do serviço. Esse mapa identifica tanto as atividades de linha de frente (que envolvem o cliente) como as atividades de retaguarda (que dão suporte à linha de frente). Segundo Brown et al. (1994), uma diferença primária entre o service blueprint e o fluxograma tradicional de operações industriais é que o blueprint incorpora o cliente e as ações do cliente no mesmo fluxograma do resto da operação.

Têm sido relatadas inúmeras aplicações para o service blueprint, como, por exemplo: na identificação dos pontos de falha do processo (SHOSTACK, 1984; CHASE \& 
STEWART, 1994; ROTONDARO \& OLIVEIRA, 1999); na identificação dos gargalos do processo, planejamento da capacidade e tempos de execução, análise de custos do processo, projeto de trabalho e melhoria da produtividade (BATESON \& HOFFMAN, 2001; LOVELOCK \& WRIGHT, 2001; TÉBOUL, 1999; LOVELOCK, 1995; SCHMENNER, 1995); no desenvolvimento de novos serviços (RAMASWAMY, 1996); na identificação dos processos-chave e áreas estratégicas de decisão (GIANESI \& CORRÊA, 1994); e na análise e definição do posicionamento estratégico de uma empresa de serviços (SHOSTACK, 1987).

é um refinamento do service blueprint, mas, mesmo assim, afirma que ele é orientado para a tarefa e não para o cliente.

Outra técnica utilizada para projetar processos de serviços é a estrutura de processamento de clientes, apresentada por Slack et al. (1997). Ao invés de mapear as atividades específicas de cada tipo de serviço, como fazem o service blueprint e o mapa do serviço, a estrutura de processamento de clientes propõe um modelo genérico de atividades-chave que são comuns à maioria dos processos de serviços. Assim, o processo do serviço pode ser projetado por meio do planejamento de cada atividade genérica em cada caso particular. É importante observar que essa técnica visa especificamente o fluxo de clientes, identificando apenas as atividades que envolvem o cliente (momentos da verdade). Porém, devido ao estabelecimento de atividades genéricas, o modelo gerado pela estrutura de processamento de

Mesmo com toda a popularidade e facilidade de uso do service blueprint, podem-se encontrar algumas limitações na técnica. Tseng et al. (1999) afirmam que pelo fato de ter a mesma estrutura do fluxograma tradicional, o service blueprint não está focalizado na descrição da completa experiência do cliente e, além disso, não é capaz de detalhar a informação sobre a experiência que o cliente tem durante a prestação do serviço. Congram \& Epelman (1995) também criticam o service blueprint, alegando que ele revela os problemas inerentes da abordagem de fluxogramas. Esses autores questionam a ambigüidade e a inconsistência no significado dos símbolos e ainda criticam os níveis de detalhe inconsistentes que ocorrem no diagrama. Para Johnston (1999b), mesmo que o service blueprint considere as interações com o cliente, muitas vezes o processo é documentado sob a perspectiva da empresa.

Uma derivação interessante do service blueprint é a técnica conhecida como mapa do serviço (KINGMANBRUNDAGE, 1991; KINGMAN-BRUNDAGE et al., 1995). O mapa do serviço tenta integrar as várias dimensões envolvidas na gestão do serviço, desde a gerência da empresa até o cliente. Para isso, ele divide os processos de serviços não somente em duas partes (linha de frente $\mathrm{e}$ retaguarda), mas em cinco diferentes zonas: zona do cliente, zona da linha de frente, zona da retaguarda, zona de suporte e zona da gerência. Mesmo com uma maior abrangência que o service blueprint no que se refere à gestão do serviço como um todo, o mapa do serviço não apresenta grandes diferenças com o service blueprint no que se refere ao projeto e análise de processos. Dessa forma, pode-se afirmar que ele possui as mesmas desvantagens do service blueprint. Johnston (1999b) considera que o mapa do serviço clientes torna-se muito distante da realidade quando se consideram processos de serviços específicos. De fato, a rigidez da estrutura de processamento de clientes torna esse diagrama incapaz de descrever com precisão a experiência do cliente.

Quando se considera apenas a análise dos processos de serviços, duas técnicas merecem destaque: a walk-throughaudit (WTA) e a análise da transação de serviço. A WTA é uma espécie de auditoria do serviço, baseada em uma série de questões dirigidas aos clientes e gerentes de serviços (FITZSIMMONS \& FITZSIMMONS, 1998; KOLJONEN $\&$ REID, 2000). A grande diferença entre a WTA e a pesquisa de satisfação do cliente, é que a WTA analisa a experiência do cliente ao longo de cada estágio do processo, ao invés de fornecer somente uma avaliação global, como nas pesquisas de satisfação (KOLJONEN \& REID, 2000). Mesmo com todas as vantagens, Johnston (1999b) afirma que a principal fraqueza da WTA é que a análise através de questões sistematicamente estruturadas pode comprometer a flexibilidade requerida para avaliar as percepções do cliente ao longo do processo.

Assim como na WTA, na análise da transação de serviço o processo é avaliado do ponto de vista do cliente. Para isso, a técnica combina quatro elementos críticos: o conceito do serviço, o processo do serviço, a avaliação da qualidade em cada transação, e a interpretação do serviço pelo cliente (JOHNSTON, 1999b). Na análise da transação de serviço, tanto a avaliação como a descrição do processo são feitas pelo cliente. Mesmo que a WTA avalie o processo segundo a percepção do cliente, esse processo é previamente mapeado pela empresa e não pelo cliente. Já na análise da transação de serviço, o cliente é quem descreve e avalia as transações, que podem ser ou não percebidas em diferentes ocasiões. Dessa 
maneira, o processo é descrito como ele realmente ocorre e como ele realmente é percebido pelo cliente.

A principal limitação, tanto da WTA quanto da análise da transação de serviço, é que ambas as técnicas somente permitem a análise do processo e não o projeto do processo. Embora a avaliação do processo seja fundamental, é interessante que o projeto e a análise estejam integrados por meio de uma única técnica, pois assim é possível utilizar uma linguagem comum no ciclo de melhoria do serviço (projeto atual / análise / melhoria do projeto atual ou novo projeto).

À medida que as técnicas de representação de processos foram evoluindo, técnicas mais sofisticadas foram sendo aplicadas em operações de serviços. Um bom exemplo é a aplicação do IDEF3, uma técnica que foi inicialmente desenvolvida para processos industriais. O IDEF3 é um dos integrantes da família de técnicas IDEF, que foi desenvolvida pela Força Aérea dos Estados Unidos com o objetivo de descrever, especificar e modelar sistemas de manufatura (PLAIA \& CARRIE, 1995). Mesmo com sua aplicação na manufatura, Tseng et al. (1999) demonstraram ser bastante viável a aplicação do IDEF3 na descrição de processos de serviços. Para isso, Tseng et al. (1999) fizeram algumas simplificações e adaptações na técnica, visando a sua utilização no caso específico das operações de serviços.

O IDEF3 adaptado por Tseng et al. (1999) considera a participação do cliente no serviço, descrevendo o processo por meio de diagramas e informações adicionais para cada atividade. A representação precisa da sequiência de atividades permite mapear a experiência que o cliente tem durante a prestação do serviço. O IDEF3 adaptado não mostra as atividades de retaguarda, como fazem o service blueprint e o mapa do serviço, pois seu foco é fluxo de clientes ao longo do processo. Uma das principais limitações da técnica, é que ela deixa a desejar no que diz respeito à avaliação de desempenho do processo.

\section{"SERVPRO"}

Com o objetivo de selecionar uma técnica que fosse adequada para o projeto e análise de processos de serviços, Santos (2000) fez uma ampla revisão e avaliação de técnicas previamente selecionadas. A avaliação de técnicas teve como base cinco requisitos: (1) Adequação tanto para o projeto quanto para a análise de processos de serviços; (2) Descrição da experiência de serviço do ponto de vista do cliente; (3) Representação gráfica baseada em diagramas; (4) Facilidade de uso; (5) Suporte para a avaliação de desempenho do processo. Na avaliação, observou-se que o IDEF3 adaptado por Tseng et al. (1999) atendia melhor aos requisitos propostos do que as outras técnicas revisadas. Porém, o IDEF3 adaptado ainda demonstrou apresentar uma deficiência no quinto requisito, pois não continha elementos que pudessem dar suporte para a avaliação de desempenho do processo.
Em busca de uma técnica que fosse adequada para serviços, foram propostas algumas alterações na técnica apresentada por Tseng et al. (1999). Essas alterações procuraram suprir as deficiências da técnica em relação aos requisitos propostos e também aumentaram a adequação da técnica aos processos de serviços. A técnica resultante foi "batizada" de SERVPRO (derivada das palavras "serviço" e "processo") e será apresentada a seguir.

\section{Aspectos gerais}

Uma das características do IDEF3 adaptado por Tseng et al. (1999) é que as atividades de retaguarda não são mostradas, mas somente as atividades de linha de frente. Essa característica foi mantida no SERVPRO. Apesar de não descrever os processos de retaguarda, essa técnica permite mapear a experiência que o cliente tem durante a prestação do serviço. A técnica parte do princípio que a melhoria das operações deve começar a partir dos problemas detectados na linha de frente e percebidos pelo cliente. Depois da constatação de que o desempenho na linha de frente está comprometendo a qualidade percebida pelo cliente, é que se procura identificar as causas dos problemas, que muitas vezes (mas nem sempre) podem ter origem nas atividades de retaguarda. Porém, o ponto de partida para a melhoria da qualidade em serviços está no cliente, o que é bastante coerente com o princípio da qualidade percebida.

Pode-se dizer que o SERVPRO tem dois elementos componentes: diagrama e documento de elaboração. $\mathrm{O}$ diagrama faz representação gráfica do processo e o documento de elaboração fornece informações adicionais para as atividades, complementando a representação do processo. O diagrama obedece ao princípio de decomposição hierárquica, onde o processo é representado inicialmente no mais alto nível de abstração, podendo ser decomposto de acordo com o nível de detalhamento requerido.

O SERVPRO utiliza o documento de elaboração somente para as atividades representadas graficamente no mais baixo nível hierárquico, e não para todas as atividades, como faz o IDEF3. Essa modificação foi feita devido à sobreposição de informações que pode ocorrer quando se utiliza o documento de elaboração para todas as atividades. Normalmente, quando se decompõe uma atividade, as informações contidas no documento de elaboração da atividade em nível mais alto passam a ser o somatório das informações dos documentos de elaboração das atividades derivadas em nível mais baixo. É como se as informações do documento de elaboração da atividade-mãe fossem divididas entre os documentos de elaboração das atividades-filhas. Por isso, torna-se desnecessária a utilização do documento de elaboração para todos os níveis de atividades. Isso simplifica ainda mais a técnica, aumentando sua facilidade de uso.

Para decidir se o documento de elaboração será feito ou 
não para uma atividade, é necessário ter em mente o nível de detalhamento requerido. No entanto, as informações do documento de elaboração também irão auxiliar na definição do nível de detalhamento. Mesmo que a representação final do processo não inclua todos os documentos de elaboração feitos durante a fase de mapeamento, muitas vezes é necessário que sejam feitos documentos de elaboração para atividades que não se tem certeza se deverão ser decompostas. A partir da análise do documento de elaboração de uma atividade, pode-se concluir se essa atividade deve ser detalhada ou não. A Figura 1 ilustra os elementos componentes do SERVPRO e a função do nível de detalhamento na ligação entre o diagrama e o documento de elaboração.

Outro fator a ressaltar, é que a estrutura gráfica dos diagramas do IDEF3 adaptado por Tseng et al. (1999) praticamente não sofreu alterações. No SERVPRO, as principais modificações foram feitas no documento de elaboração. Além de procurar melhorar a adequação das informações do documento de elaboração aos processos de serviços, procurou-se introduzir informações com o objetivo de suprir a deficiência do IDEF3 adaptado por Tseng et al. (1999) no suporte para a avaliação de desempenho do processo. Para isso, procurou-se relacionar os determinantes da qualidade em serviços com as medidas de desempenho para cada atividade do processo.

Figura 1: Elementos componentes do SERVPRO.

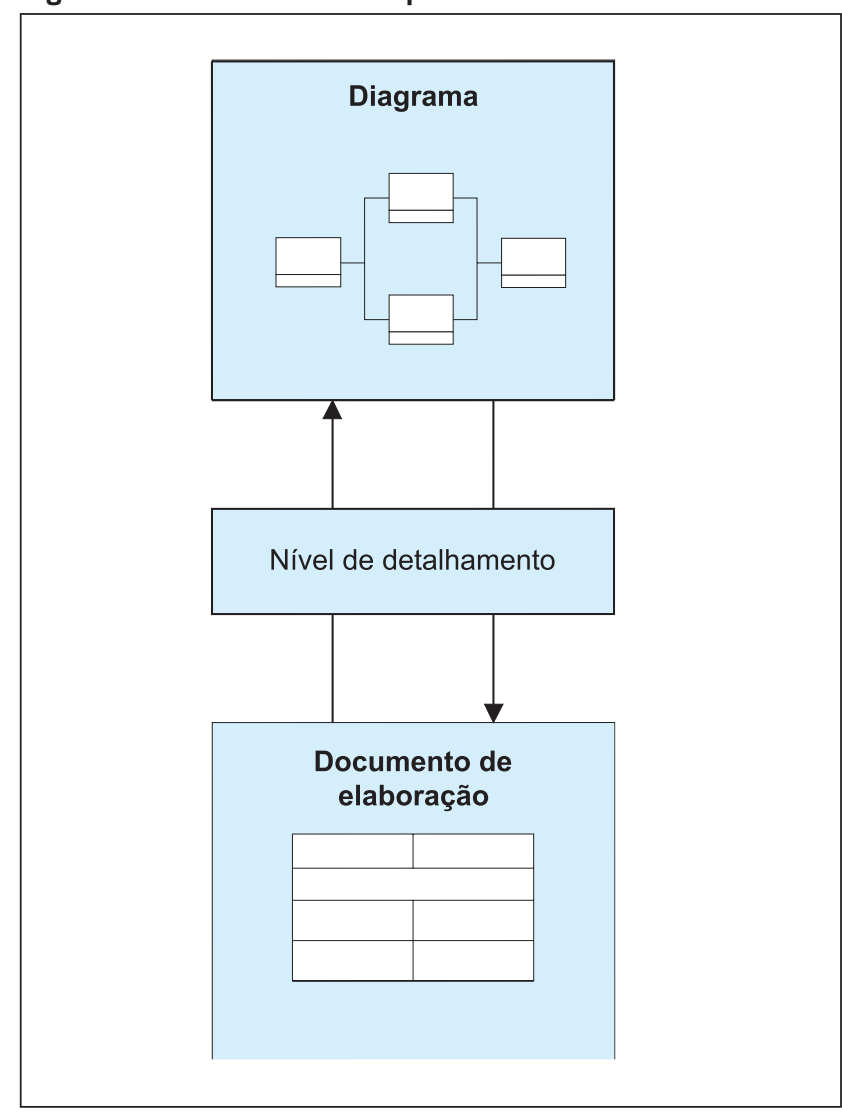

\section{Diagrama}

Os elementos básicos que compõem os diagramas são: atividades de interação, setas, junções e "Go-to".

Atividades de interação. As atividades do processo receberam o nome de atividades de interação, devido ao aspecto de interação com o cliente em cada atividade. Elas são representadas por retângulos em que constam o nome e o número de cada atividade. O nome da atividade que é apresentado no diagrama pode ser chamado de rótulo, sendo que ele pode ser uma abreviatura do nome completo, por questões de apresentação gráfica. Normalmente, prefere-se que o nome da atividade seja iniciado com um verbo seguido por substantivo(s), para transmitir uma noção de ação do cliente. O número de cada atividade de interação irá depender do nível de detalhamento da atividade, de acordo com o princípio de decomposição hierárquica (Figura 2).

Setas. As setas são usadas para conectar as atividades de interação, indicando o fluxo do processo e a seqüência em que as atividades ocorrem. As setas podem também ser chamadas de ligações de precedência, pois quando duas atividades são conectadas por uma seta a ocorrência da primeira precede a ocorrência da segunda.

Junções. As junções são mecanismos utilizados para indicar as ramificações do processo. O fluxo do processo pode convergir ou divergir em atividades que ocorrem paralelamente ou alternativamente. As junções do diagrama são assíncronas, isto é, as atividades paralelas não precisam começar ou terminar ao mesmo tempo. O SERVPRO utiliza os seguintes tipos de junções:

- Junção \& divergente: quando todas as atividades após a junção ocorrem paralelamente.

- Junção O divergente: quando uma ou mais atividades após a junção ocorrem.

- Junção X divergente: quando somente uma atividade após a junção ocorre.

- Junção \& convergente: quando todas as atividades que terminam na junção ocorrem paralelamente.

- Junção O convergente: quando uma ou mais atividades que terminam na junção ocorrem.

- Junção X convergente: quando somente uma atividade que termina na junção ocorre.

As junções convergentes ou divergentes são representadas por retângulos menores que os das atividades de interação, em que constam o número da junção e o tipo da junção (Figura 3). O número da junção está relacionado com o nível de detalhamento em que ela se encontra, que será o número da atividade que está sendo decomposta (ou seja, do nível acima) seguido do número de ordem em que a junção aparece.

Para uma melhor compreensão do significado das jun- 
ções e de sua importância na descrição do fluxo do processo, é interessante que se considerem alguns exemplos:

- Exemplo 1 (junção \&): Uma lanchonete fast-food estava recebendo constantes reclamações quanto ao tempo de espera na fila. Para amenizar o problema, resolveu colocar funcionários recolhendo os pedidos dos clientes na fila. Quando o cliente chegava ao caixa, seu lanche já estava pronto e ele só precisava pagar. Com essa ação, a empresa reduziu a percepção do cliente do tempo de espera, aumentou a eficiência do processo e eliminou a atividade "aguardar o lanche" (que não agrega nenhum valor para o cliente).
A Figura 4 mostra a mudança no diagrama do processo que abrange da atividade "aguardar na fila" até a atividade "receber o lanche". A atividade "fazer o pedido e pagar" foi desagregada: "fazer o pedido" passou a acontecer em paralelo com "aguardar na fila" e o pagamento passou a acontecer agregado à atividade "receber o lanche". O diagrama anterior, que tinha todas as atividades ocorrendo em série, passou a ter duas atividades ocorrendo em paralelo, o que só pode ser representado com a utilização da junção \&. A primeira junção no processo modificado é divergente e a segunda é convergente.
Figura 2: Decomposição de uma atividade de interação.

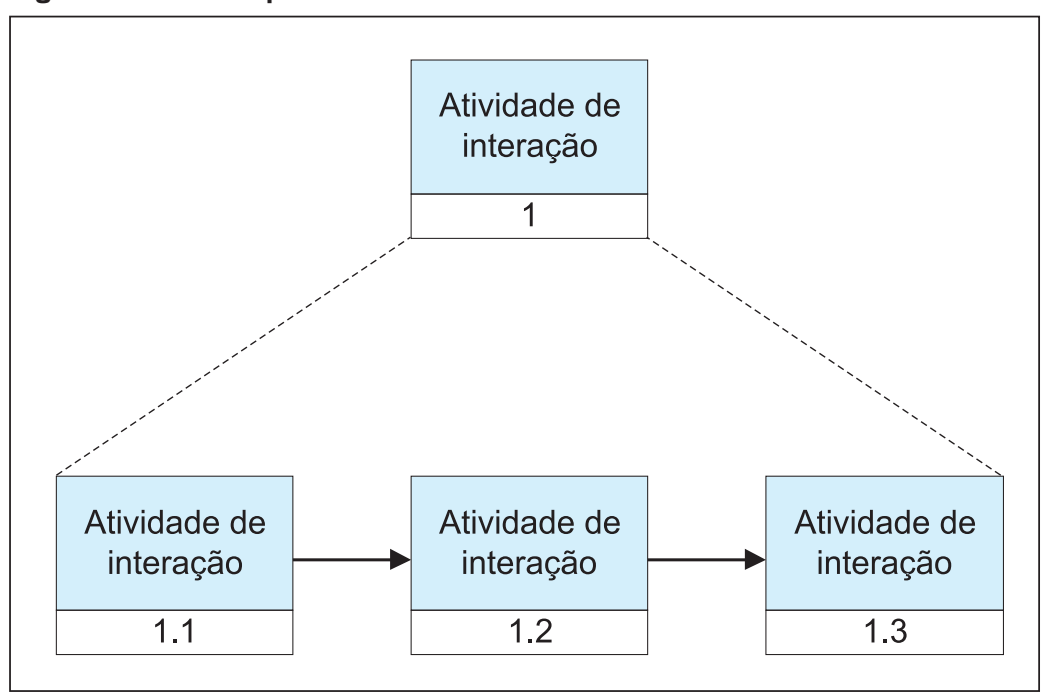

Figura 3: Junções do diagrama.

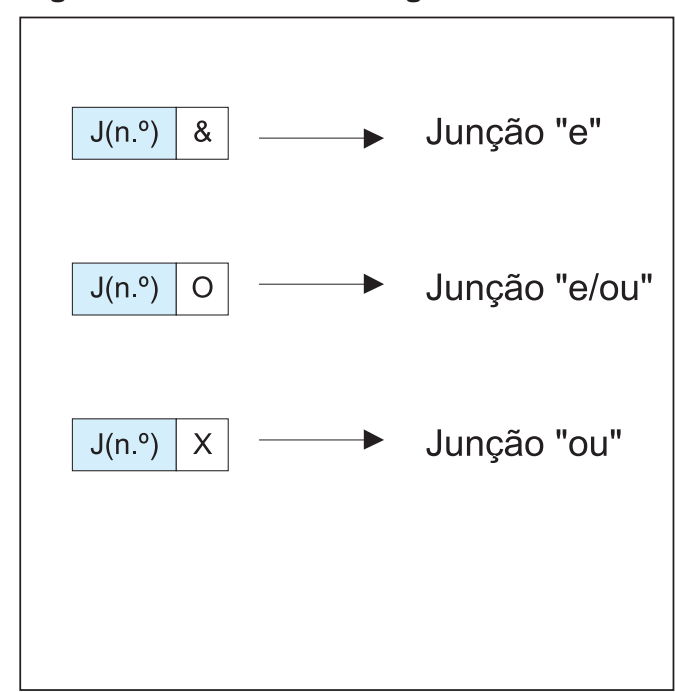

Figura 4: A junção \& permite representar fluxos paralelos no processo.

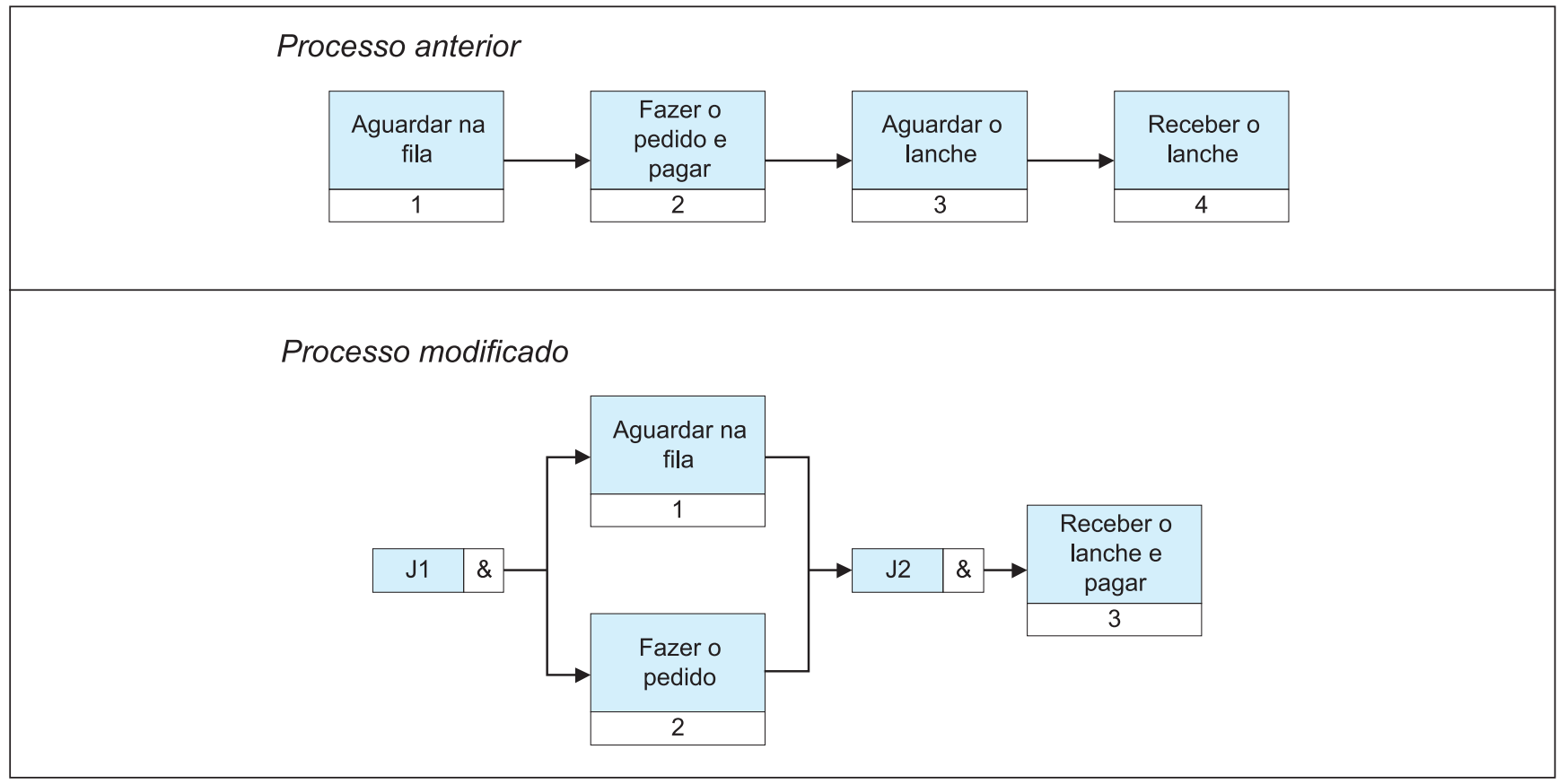


- Exemplo 2 (junções $\mathbf{X}$ e $\mathbf{O}$ ): Um restaurante tipo buffet possui três ambientes diferentes: massas (cozinha italiana), comida chinesa e frutos do mar. O restaurante estabelece seus preços por pessoa, de acordo com o ambiente escolhido. Assim, o cliente tem que fazer uma opção antes de se servir. No processo do restaurante, a atividade de interação "servir-se" pode ser decomposta nas três opções, que ocorrem alternativamente no processo. O diagrama mostrado na Figura 5 representa o processo desde a atividade "entrar no restaurante" até a atividade "sentar-se à mesa", sendo que a junção $X$ é usada para indicar os fluxos alternativos no processo. Se a gerência do restaurante perceber que seus clientes gostariam de experimentar mais de uma opção na mesma refeição, poderá estipular um preço único, permitindo o livre acesso em todos os ambientes. Para representar o fluxo dos clientes nesse caso, basta substituir a junção $\mathrm{X}$ pela junção $\mathrm{O}$, pois ela permite representar fluxos alternativos não excludentes que ocorrem no processo. Isso significa que o cliente poderá se servir de uma ou mais opções oferecidas.

Go-to. É um elemento que auxilia a descrição de fluxo quando há necessidade de indicar uma ligação com a próxima ocorrência no processo e quando essa ocorrência não está conectada por uma seta (a própria tradução do inglês já explica: "vá-para"). Quando o Go-to se refere a uma atividade de interação, significa que a próxima ocorrência no processo será a atividade referenciada. Quando o Go-to se refere a uma junção, significa que as próximas ocorrências no processo serão as atividades que acontecem após a junção referenciada. Muitas vezes, o Go-to indica a possibilidade da ocorrência de um looping no processo, ou seja, a possibilidade do fluxo de processo voltar a um ponto anterior do processo. O Go-to é representado por um retângulo com o nome "Go-to" seguido do número da atividade ou junção referenciada.

No exemplo do restaurante da Figura 5, é necessário considerar a possibilidade do cliente se servir novamente quantas vezes desejar. O Go-to pode então ser usado para indicar a possibilidade de o cliente repetir a atividade "servir-se". A Figura 6 mostra o digrama do processo da junção J1 até a atividade "pagar a conta". Nesse caso, considerou-se que o cliente poderia se servir dos três tipos de comida.

\section{Documento de elaboração}

O documento de elaboração fornece informações adicionais para as atividades de interação. Como mencionado anteriormente, o documento de elaboração é feito somente para as atividades de interação representadas no mais baixo nível hierárquico. $\mathrm{O}$ documento de elaboração do SERVPRO tem o formato mostrado na Figura 7, contendo as seguintes informações: nome, número e rótulo da atividade de interação, elemento(s) de contato, interações entre $\mathrm{o}(\mathrm{s})$ elemento(s) e o cliente, operações do(s) elemento(s), operações do cliente, determinantes da qualidade e medidas de desempenho.

Elemento(s) de contato. Os elementos de contato re-

Figura 5: A junção X permite representar fluxos alternativos no processo.

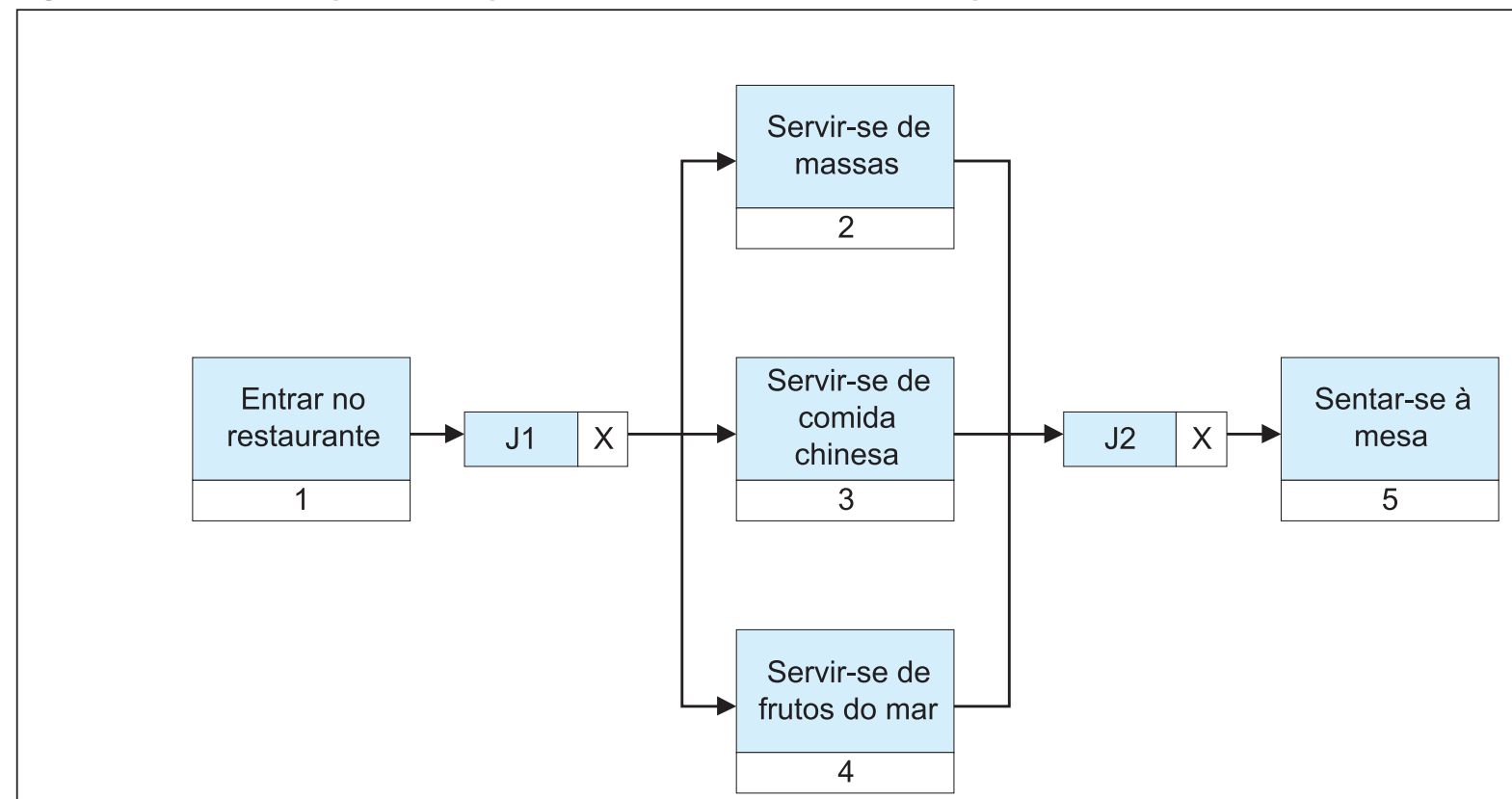


presentam todos os recursos identificáveis em cada atividade de interação, que entram em contato com o cliente e que podem afetar sua percepção da qualidade do serviço. Esses elementos podem ser instalações, bens facilitadores, funcionários, software, ou seja, tudo que pode afetar a percepção do cliente na atividade de interação. A Figura 8 apresenta os elementos de contato da atividade "pagar a conta" do processo do restaurante da Figura 6.
Interações entre o(s) elemento(s) e o cliente. Nesta seção são representadas as interações que ocorrem em cada atividade de interação, que podem se dar no contato pessoal ou com o ambiente inanimado. Freqüientemente, as interações entre os elementos e o cliente correspondem a subatividades da atividade de interação que não foram representadas graficamente. Outras vezes, as interações correspondem a operações dos elementos de contato ou

Figura 6: 0 Go-to indica a possibilidade de o cliente voltar a uma atividade anterior.

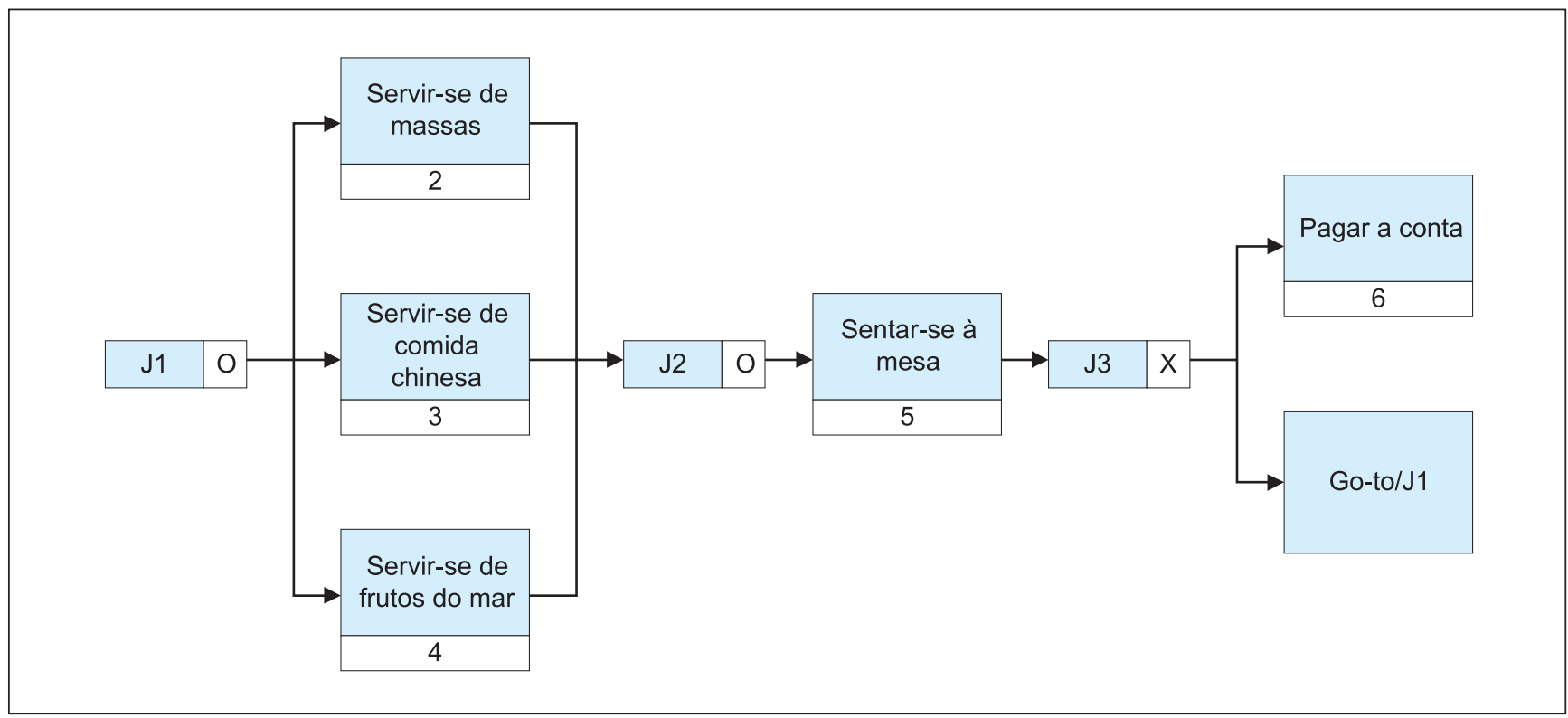

Figura 7: Documento de elaboração.

\begin{tabular}{|l|l|}
\hline $\begin{array}{l}\text { Atividade de interação №: } \\
\text { Nome: }\end{array}$ & Elemento(s) de contato: \\
Rótulo: & Operações do cliente: \\
\hline Interações entre o(s) elemento(s) e o cliente: & \\
\hline Operações do[s] elemento(s): & Medidas de desempenho: \\
\hline Determinantes da qualidade: & \\
\hline & \\
\hline
\end{tabular}


operações do cliente. Porém, o objetivo dessas informações é representar as relações de troca entre o cliente e o sistema de operações de serviços. Uma ação do cliente pode corresponder a uma reação do sistema de serviços, ou vice-versa. As interações são representadas com os seguintes símbolos:

$\rightarrow$ Ação do cliente.

$\leftarrow$ Reação do cliente.

IIII $\rightarrow$ Ação do sistema de serviços.

$\leftarrow$ 亚 Reação do sistema de serviços.
A Figura 8 mostra as interações entre os elementos e o cliente da atividade "pagar a conta" da Figura 6.

Operações do(s) elemento(s). As operações desempenhadas pelos elementos de contato com o cliente são as operações necessárias para a produção do serviço que são feitas para o cliente, em cada atividade de interação.

Operações do cliente. Algumas vezes o cliente participa como co-produtor do serviço, realizando operações que poderiam ser feitas pela empresa. As operações do cliente são as operações necessárias para a produção do serviço que são feitas pelo cliente, em cada atividade de interação.

Figura 8: Elementos de contato e interações da atividade "pagar conta".

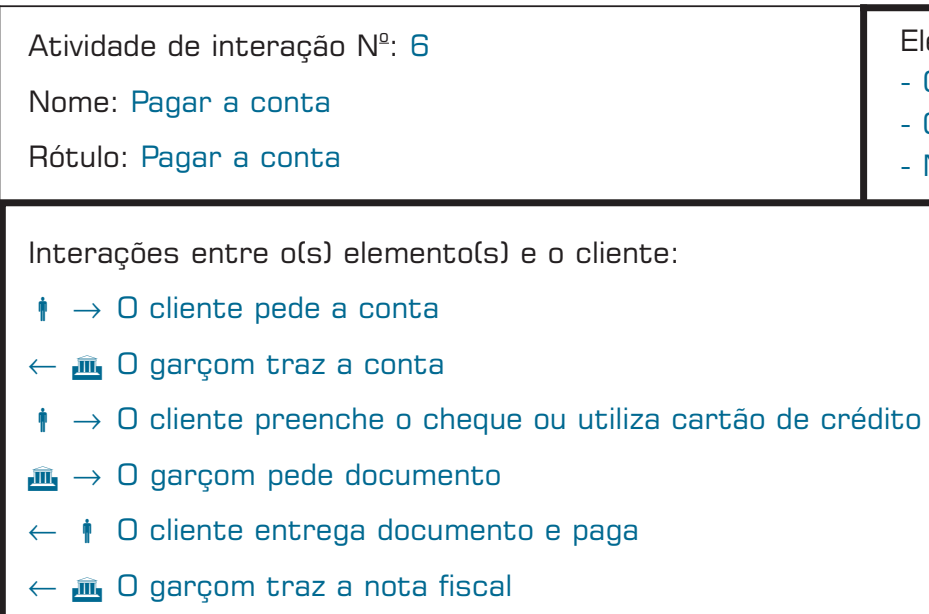

Figura 9: Operações do[s] elemento[s] e operações do cliente da atividade "pagar a conta".

\begin{tabular}{|c|c|}
\hline $\begin{array}{l}\text { Atividade de interação №: } 6 \\
\text { Nome: Pagar a conta } \\
\text { Rótulo: Pagar a conta }\end{array}$ & $\begin{array}{l}\text { Elemento[s] de contato: } \\
\text { - Garçom } \\
\text { - Conta } \\
\text { - Nota fiscal }\end{array}$ \\
\hline \multicolumn{2}{|c|}{ 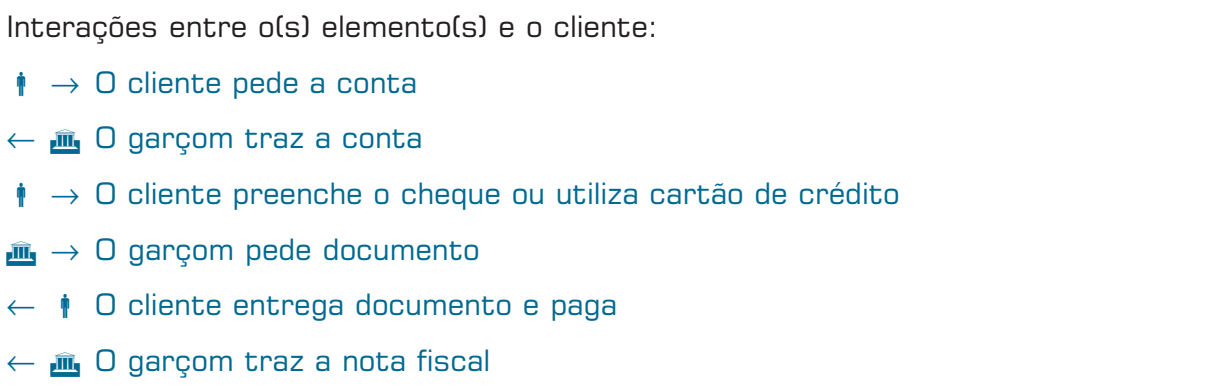 } \\
\hline $\begin{array}{l}\text { Operações do(s) elemento(s): } \\
\text { - Garçom: trazer a conta } \\
\text { - Garçom: receber pagamento e entregar a nota }\end{array}$ & $\begin{array}{l}\text { Operações do cliente: } \\
\text { - Preencher o cheque }\end{array}$ \\
\hline
\end{tabular}


Continuando a construção do documento de elaboração, a Figura 9 mostra as operações do(s) elemento(s) e as operações do cliente que ocorrem na atividade "pagar a conta" da Figura 6.

Determinantes da qualidade. Cada atividade de interação terá determinantes da qualidade em serviços que são considerados críticos para a atividade. Esses determinantes também são representados no documento de elaboração, sendo que geralmente estão relacionados com os elementos de contato, com as interações entre os elementos e o cliente, com as operações dos elementos e com as operações do cliente. Os determinantes da qualidade representam os parâmetros em que a qualidade do serviço é avaliada pelo cliente.

Medidas de desempenho. Para cada determinante da qualidade apresentado no documento de elaboração, podese sugerir medidas correspondentes que irão indicar o desempenho das operações de serviços em cada atividade de interação. Assim, as informações do documento de elaboração, além de sinalizar os determinantes predominantes em cada atividade, permitem fornecer suporte para a avaliação de desempenho em relação a cada determinante. A Figura 10 traz os determinantes da qualidade e as respectivas medidas de desempenho para a atividade "pagar a conta" da Figura 6, completando, assim, o documento de elaboração para essa atividade.

\section{Nivel de detalhamento}

A decomposição hierárquica de cada atividade permite que se obtenha qualquer nível de detalhamento, de acordo com a complexidade do processo. No entanto, nem sempre é viável ou necessário detalhar ao máximo todas as atividades de interação. Na fase de análise de processos, o nível de detalhamento está associado ao grau de melhoria desejado, o que não necessariamente significa que um detalhamento excessivo traga o retorno esperado. De acordo com Soliman (1998), existe um ponto em que não se obtém mais informações úteis com mapeamento de processos. Isso significa que há um momento em que o benefício alcançado com o mapeamento não justifica o custo do detalhamento excessivo.

Pode-se definir o nível de detalhamento ideal com base em vários fatores. Harrington et al. (1997) afirmam que o nível de detalhe adequado depende dos objetivos do mapeamento de processos em cada situação. No projeto de um

Figura 10: Determinantes da qualidade e medidas de desempenho para a atividade "pagar a conta".

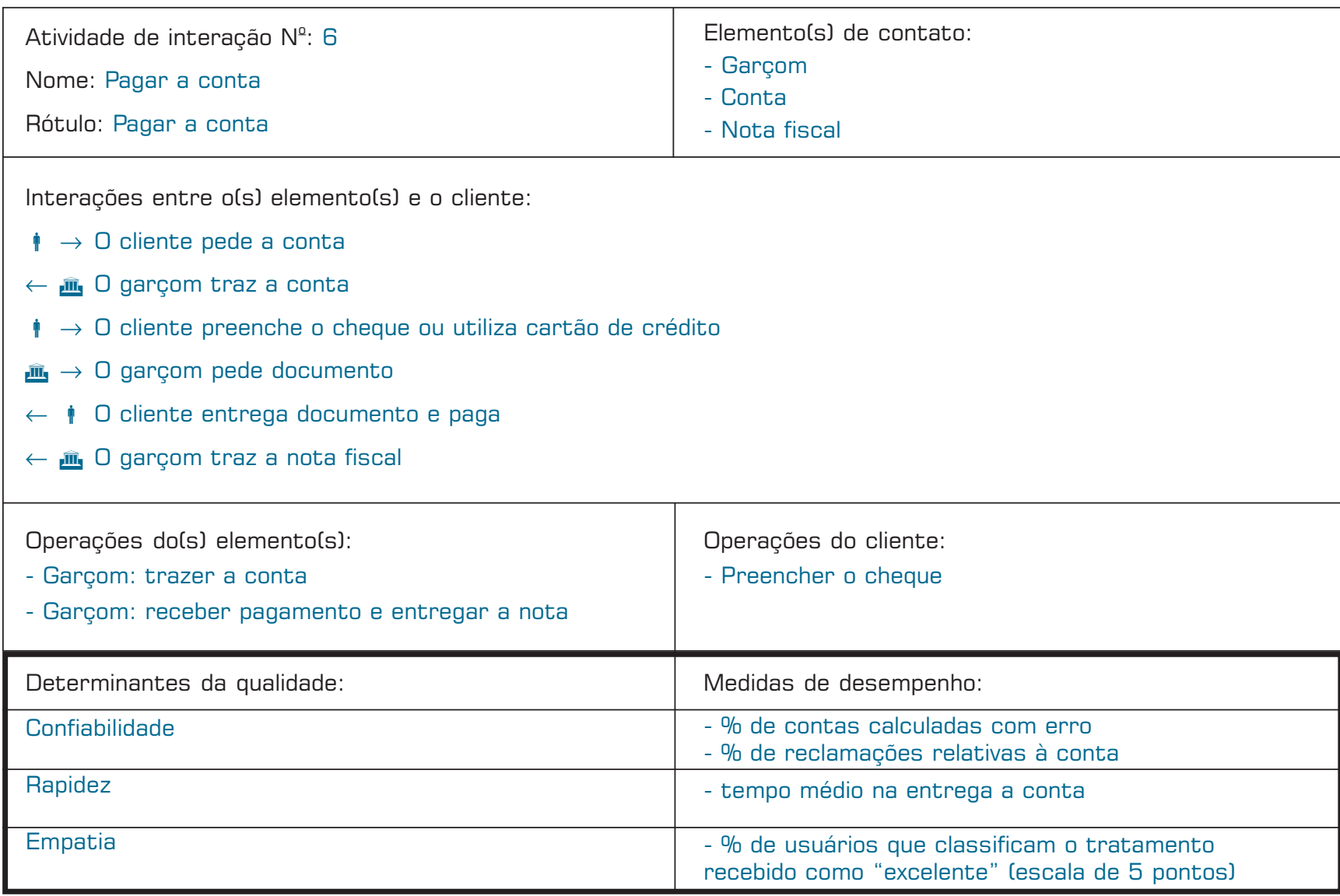


novo processo, nem sempre se requer o mesmo nível de detalhamento do que na análise de processos existentes. Mesmo que isso não seja uma regra, a análise de processos existentes pode requerer um nível de detalhamento maior, principalmente quando se quer identificar problemas através do profundo conhecimento do processo.

Soliman (1998) baseia-se nos custos para definir o nível de detalhamento, estabelecendo um nível ótimo de detalhamento que corresponde ao mínimo custo no mapeamento de processos. $\mathrm{O}$ autor considera que existem custos de mapeamento, que aumentam à medida que o nível de detalhe aumenta. Esses custos estão relacionados ao esforço e tempo despendidos ao coletar, analisar e interpretar as informações. Ao mesmo tempo, Soliman (1998) afirma que um processo projetado com baixo nível de detalhe também representa custos, que diminuem à medida que o nível de detalhe aumenta. Esses custos estão relacionados à utilização de operadores com maior qualificação (para conseguir entender e operar processos pouco detalhados) e aos erros e defeitos que ocorrem com maior facilidade em um processo pouco detalhado (a exatidão na execução depende do nível de detalhe em que o processo foi projetado). Considerando o balanceamento entre os custos di- bém podem auxiliar bastante ao se definir o nível de detalhe adequado. Assim, algumas sugestões podem ser levadas em consideração. Em geral, não é mais necessário detalhar uma atividade quando:

- As informações dos documentos de elaboração das atividades derivadas no nível inferior (atividades-filhas) são muito simples ou elementares.

- As informações dos documentos de elaboração de diferentes atividades derivadas são iguais ou muito semelhantes, ou seja, quando os documentos de elaboração das atividades-filhas não apresentam diferenças significativas.

- O documento de elaboração da atividade a detalhar apresenta informações muito elementares.

- A atividade a detalhar apresenta uma única interação entre o(s) elemento(s) e o cliente.

\section{CONSIDERAC̣̃̃ES FINAIS}

O SERVPRO representa uma técnica bastante usual para o projeto e gestão de operações de serviços. Os recursos gráficos do diagrama permitem descrever facilmente a experiência do cliente no serviço, considerando a característica de participação do cliente e a variabilidade dos processos de serviços. Até mesmo os processos mais complexos podem ser representados com simplicidade, inicialmente de uma forma mais agregada (nível macro), aumentando o detalhamento de

retamente proporcionais e os custos inversamente proporcionais ao nível de detalhamento, pode-se obter um ponto ótimo que minimiza o custo total de mapeamento. Soliman (1998) estabeleceu um modelo de programação linear que calcula essa otimização.

Entretanto, o modelo de Soliman (1998) não inclui benefícios do detalhamento de processos que não são facilmente mensuráveis. Esses benefícios podem ser avaliados por gerentes com experiência e senso prático. Por isso, a experiência do analista ou projetista de processos é um fator fundamental na definição do nível de detalhamento adequado. Outro fator que Soliman (1998) não considerou, é que processos diferentes podem ter níveis de detalhamento diferentes. Especialmente em processos de serviços, que tendem a ter uma variabilidade maior, não é necessário estabelecer o mesmo nível de detalhamento para processos com graus de complexidade e necessidades de melhoria diferentes.

Mesmo existindo diferentes critérios para definir o nível de detalhamento no projeto e análise de processos, as informações do documento de elaboração do SERVPRO tam- acordo com a necessidade (nível micro).

O documento de elaboração apresenta alterações significativas em relação à adaptação original de Tseng et al. (1999). Ao invés de "atributos do objeto de contato", o SERVPRO sugere a identificação dos determinantes da qualidade em serviços e medidas de desempenho correspondentes em cada atividade de interação. Isso dá uma contribuição muito mais realista para a melhoria e garantia da qualidade em serviços do que simplesmente identificar atributos que nem sempre são significativos para a gestão do serviço. Outra alteração importante, foi a separação entre "operações do(s) elemento(s)" e "operações do cliente", o que facilita a análise das operações que poderiam ser melhor desempenhadas pela empresa ou pelo cliente. A definição mais precisa no significado das "interações entre o(s) elemento(s) e o cliente" do documento de elaboração do SERVPRO consegue suprir as informações que não eram explicitadas nas "relações entre o objeto e o cliente" da proposta de Tseng et al. (1999). 
O SERVPRO representa uma evolução da idéia inicial de Tseng et al. (1999) e um refinamento da adaptação do IDEF3 aos processos de serviços. Ainda assim, é possível fazer algumas sugestões:

- Além de visar a melhoria da qualidade, o SERVPRO poderia ser adaptado para outras finalidades, como, por exemplo, automação de processos e análise de custos dos processos.

- Recomenda-se que o SERVPRO seja aplicado também nos processos de retaguarda, fazendo as devidas adaptações se necessário. É interessante que seja explorada a relação dos processos de linha de frente com os processos de retaguarda, analisando a influência dessa interfa- ce na qualidade dos serviços. Além disso, poderiam ser analisados os fluxos de materiais e informações, e não somente o fluxo de clientes no processo.

- É interessante que seja verificada a aplicabilidade do SERVPRO em diferentes organizações. Assim, podem-se verificar as possíveis implicações na aplicação da técnica em diferentes tipos de processos de serviços.

- O SERVPRO também pode servir de base para possíveis aplicações computacionais. Um software poderia ser desenvolvido de forma a otimizar a utilização da técnica e possibilitar novas aplicações, como, por exemplo, na simulação computacional dos processos.
BATESON, John E. G.; HOFFMAN, K. Douglas. Marketing de serviços. 4. ed. Porto Alegre: Bookman, 2001 $495 \mathrm{p}$.

BROWN, Stephen W., FISK, Raymond P., BITNER, Mary Jo. The development and emergence of services marketing thought. International Journal of Service Industry Management. UK, v. 5, n. 1, p. 21-48, 1994

CHASE, Richard B., STEWART, Douglas M. Make your service fail-safe. Sloan Management Review. USA, v. 35, n. 3, p. 35-44, spring, 1994.

CONGRAM, Carole, EPELMAN, Michael. How to describe your service: an invitation to the structured analysis and design technique. International Journal of Service Industry Management. UK, v. 6, n. 2, p. 6-23, 1995.

FITZSIMMONS, James A., FITZSIMMONS, Mona J. Service management: operations, strategy, and information technology. 2.ed. USA: Irwin/McGraw-Hill, 1998. $613 \mathrm{p}$.

GIANESI, Irineu G. N., CORRÊA, Henrique Luiz. Administração estratégica de serviços: operações para a satisfação do cliente. São Paulo: Atlas, 1994. $233 \mathrm{p}$.

GUMMESSON, Evert. Service management: an evaluation and the future. International Journal of Service Industry Management. UK, v. 5, n. 1, p. 77-96, 1994
HARRINGTON, H. James, ESSELING, Erik K. C., NIMWEGEN, Harm van. Business process improvement workbook: documentation, analysis, design, and management of business process improvement. USA: McGraw-Hill, 1997. $314 \mathrm{p}$.

JOHNSTON, Robert. Operations: from factory to service management. International Journal of Service Industry Management. UK, v. 5, n. 1, p. 49-63, 1994.

. Service operations management: return to roots. International Journal of Operations \& Production Management. UK, v. 19, n. 2, p. 104-124, 1999a.

Service transaction analysis: assessing and improving the customer's experience. Managing Service Quality. UK, v. 9 , n. 2 , p. $102-109,1999$ b.

KINGMAN-BRUNDAGE, Jane. Technology, design and service quality. International Journal of Service Industry Management. UK, v. 2, n. 3, p. 47-59, 1991

KINGMAN-BRUNDAGE, Jane, GEORGE, William R., BOWEN, David E. "Service logic": achieving service system integration. International Journal of Service Industry Management. UK, v. 6, n. 4, p. 20-39, 1995.
KOLJONEN, Elsa Lai-Ping Leong, REID, Richard A. Walk-through audit provides focus for service improvements for Hong Kong law firm. Managing Service Quality. UK, v. 10, n. 1, p. 32-45, 2000.

LOVELOCK, Christopher H. Product plus: produto + serviço $=$ vantagem competitiva. São Paulo: Makron Books, 1995. $476 \mathrm{p}$

LOVELOCK, Christopher; WRIGHT, Lauren. Serviços: marketing e gestão. São Paulo: Saraiva, 2001. 416 p.

PLAIA, Antonella, CARRIE, Allan. Application and assessment of IDEF3 process flow description capture method. International Journal of Operations \& Production Management. UK, v. 15 , n. 1 , p. $63-73,1995$.

RAMASWAMY, Rohit. Design and management of service processes: keeping customers for life. USA: Addison-Wesley, 1996. $424 \mathrm{p}$.

ROTONDARO, Roberto Gilioli, OLIVEIRA, Claudio Lopes de. O indicador do potencial de falha como ferramenta de melhoria da qualidade do serviço. In: Encontro Nacional de Engenharia de Produção, 19., Rio de Janeiro - RJ, 1999. Anais... Rio de Janeiro: UFRJ, 1999. CDROM.
SANTOS, Luciano Costa. Projeto e análise de processos de serviços: avaliação de técnicas e aplicação em uma biblioteca. Florianópolis, 2000. 110 f. Dissertação (Mestrado em Engenharia de Produção) - Universidade Federal de Santa Catarina.

SCHMENNER, Roger W. Service operations management. USA: Prentice Hall, 1995. 406 p.

SHOSTACK, G. Lynn. Designing services that deliver. Harvard Business Review. USA, v. 62 , n. 1, p. 133-139, januaryfebruary, 1984

Service positioning through structural change. Journal of Marketing. USA, v. 51, n. 1, p. 34-43, january, 1987.

SLACK, Nigel, et al. Administração da produção. São Paulo: Atlas, 1997. 726 p.

SOLIMAN, F. Optimum level of process mapping and least cost business process re-engineering. International Journal of Operations \& Production Management. UK, v. 18 , n. $9 / 10$, p. $810-816,1998$.

TÉBOUL, James. A era dos serviços: uma nova abordagem de gerenciamento. Rio de Janeiro: Qualitymark Ed., 1999. $295 \mathrm{p}$

TSENG, Mitchell M., QINHAI, Ma, SU, Chuan-Jun. Mapping customers service experience for operations improvement. Business Process Management Journal. UK, v. 5, n. 1, p. 5064, 1999. 